\title{
Feedback design using nonsmooth control Lyapunov functions: A numerical case study for the nonholonomic integrator
}

\author{
Philipp Braun ${ }^{1,2}$, Lars Grüne ${ }^{1}$, Christopher M. Kellett ${ }^{2}$
}

\begin{abstract}
Theoretical results for the existence of (nonsmooth) control Lyapunov functions (CLFs) for nonlinear systems asymptotically controllable to the origin or a closed set have been available since the late 1990s. Additionally, robust feedback stabilizers based on such CLFs have also been available though, to the best of our knowledge, these stabilizers have not been implemented. Here, we numerically investigate the properties of the closed loop solutions of the nonholonomic integrator using three control techniques based on the knowledge of two different nonsmooth CLFs. In order to make the paper self-contained, we review theoretical results on the existence of nonsmooth CLFs.
\end{abstract}

\section{INTRODUCTION}

Control Lyapunov functions (CLFs) were first introduced by Artstein in [1] and have been widely used for the design of feedback stabilizers; see, e.g., [12], [17]. However, there is a known impediment to the existence of continuously differentiable CLFs [2], [6] for systems asymptotically controllable to the origin. The existence of nonsmooth CLFs under the assumption of asymptotic controllability was first addressed by Sontag [18], wherein a continuous CLF was presented. Subsequently, existence results and, in fact, feedback stabilizers were presented in [5], [15], [9], [10]. This is an endeavour with a rich history and a comprehensive survey can be found in [4].

Generally speaking, the results above have been restricted to theoretical development without investigating the implementation of the proposed (sample and hold) feedback stabilizers, with an exception being the work of Nakamura et al. [11], [16], [14], where piecewise continuous stabilizers are developed in contrast to the piecewise constant sample and hold feedbacks considered here.

In part, this lack of attention is due to the significant theoretical and practical advances in model predictive control techniques. Since, in general, model predictive control is easy to implement (if questions like robustness and stability of the closed loop system are put aside), and feedback design based on nonsmooth CLFs can only be implemented if a CLF is known, CLF-based feedback design has not received recent attention. However, such designs have tangible benefits in the form of guaranteed closed-loop stability as well as inherent robustness properties [8].

\footnotetext{
The authors are supported by the Australian Research Council (Grant number: G1500106)

${ }^{1} \mathrm{P}$. Braun and L. Grüne are with the Mathematical Institute, Universität Bayreuth, 95440 Bayreuth, Germany, e-mail: \{philipp.braun, lars.gruene \} @ uni-bayreuth.de.

${ }^{2}$ P. Braun and C. M. Kellett are with the School of Electrical Engineering and Computing at the University of Newcastle, Callaghan, New South Wales 2308, Australia, e-mail: \{chris.kellett, philipp.braun\} @ newcastle.edu.au.
}

In this paper, we investigate the performance of the closedloop solutions for the nonholonomic integrator

$$
\left(\begin{array}{ccc}
\dot{x}_{1} & \dot{x}_{2} & \dot{x}_{3}
\end{array}\right)^{T}=\left(\begin{array}{ccc}
u_{1} & u_{2} & x_{1} u_{2}-x_{2} u_{1}
\end{array}\right)^{T},
$$

$x \in \mathbb{R}^{3}, u \in \mathbb{R}^{2}$. We propose two CLFs, with differing regularity properties, and investigate the behavior of three different feedback stabilizers based on these CLFs. The nonholonomic integrator is of particular interest since Brockett's condition implies that there exists no continuous control law asymptotically stabilizing the origin, even though the system is asymptotically controllable to the origin [2]. Thus, the nonholonomic integrator fails to admit a smooth CLF which explains the necessity of the consideration of nonsmooth CLFs and the introduction of generalized derivatives such as the Dini derivative. Additionally, the dynamics of the nonholonomic integrator are particularly interesting since they can be used to model the dynamics of mobile robots (see for example [11], [19], and the references therein). In this context, model predictive control has been applied to the nonholonomic integrator in [7], [19], and [13], for example. Here, the difficulty lies in the definition of an appropriate running cost and a prediction horizon that guarantees stability - a problem which is still not fully understood.

The paper is organized as follows. In Section II the mathematical setting is introduced. This includes definitions and properties of the dynamical systems considered, necessary and sufficient conditions for existence of (nonsmooth) CLFs, and semiconcavity, a property of importance for nonsmooth CLFs. In Section III we present three feedback design techniques based on the knowledge of a CLF. The numerical properties of these methods are analyzed in Section IV using two nonsmooth CLFs for the dynamics of the nonholonomic integrator. The paper ends with conclusions in Section V.

\section{CLFS: THEORY AND DEFINITIONS}

In this section the mathematical setting and the notations used throughout the paper are introduced. In the second part of this section necessary and sufficient conditions for the existence of (nonsmooth) CLFs based on the Dini derivative are reviewed. The section concludes with the definition and illustration of semiconcavity in the context of CLFs.

\section{A. Mathematical setting}

The system dynamics of a nonlinear time-invariant control system, including the dynamics of the nonoholonomic integrator, are described by

$$
\dot{x}=f(x, u)
$$


where $x \in \mathbb{R}^{n}$ denotes the state, $u \in \mathbb{U} \subset \mathbb{R}^{m}$ denotes the input, and $f: \mathbb{R}^{n} \times \mathbb{U} \rightarrow \mathbb{R}^{n}$ is a continuous function defining the dynamics of the system. Alternatively, the system dynamics can be represented using a differential inclusion

$$
\dot{x} \in F(x)
$$

where $F: \mathbb{R}^{n} \rightrightarrows \mathbb{R}^{n}$. Here, $F$ is defined as

$$
F(x)=\overline{\operatorname{co}}\left\{z \in \mathbb{R}^{n} \mid z=f(x, u), u \in \mathbb{U}\right\},
$$

where $\overline{\mathrm{co}}(\cdot)$ denotes the closure of the convex hull. In the context of CLFs, (2) and (3) can be used interchangeably.

We make use of the following definitions and notations. $B_{r}(x)=\left\{y \in \mathbb{R}^{n} \mid\|x-y\|<r\right\}$ denotes an open ball of radius $r>0$ centered around $x \in \mathbb{R}^{n}$ and $\bar{B}_{r}(x)=$ $\left\{y \in \mathbb{R}^{n} \mid\|x-y\| \leq r\right\}$ denotes its closure. A function $\phi$ : $\mathbb{R}_{\geq 0} \times \mathbb{R}^{n} \rightarrow \mathbb{R}^{n}$ is said to be a solution of the differential inclusion (3) from initial condition $x \in \mathbb{R}^{n}$ if it is absolutely continuous and satisfies $\dot{\phi}(t, x) \in F(\phi(t, x))$ for almost all $t \in \mathbb{R}_{\geq 0}$. We use $\phi(\cdot, x, u)$ to emphasize a particular input. $\mathcal{S}(x)$ denotes the set of solutions starting at $x$. A function $\alpha: \mathbb{R}_{\geq 0} \rightarrow \mathbb{R}_{\geq 0}$ is said to be of class $\mathcal{K}_{\infty}\left(\alpha \in \mathcal{K}_{\infty}\right)$ if $\alpha$ is continuous, strictly increasing, $\alpha(0)=0$ and $\alpha(r) \rightarrow \infty$ for $r \rightarrow \infty$. A function $\beta: \mathbb{R}_{\geq 0}^{2} \rightarrow \mathbb{R}_{\geq 0}$ is said to be of class $\mathcal{K} \mathcal{L}(\beta \in \mathcal{K} \mathcal{L})$ if $\beta(\cdot, t) \in \mathcal{K}_{\infty}$ for all $t \in \mathbb{R}_{\geq 0}$ and $\beta(r, \cdot)$ is continuous, decreasing and $\beta(r, t) \rightarrow 0$ for $t \rightarrow \infty$ for all $r \in \mathbb{R}_{>0}$. A function $\rho: \mathbb{R}_{\geq 0} \rightarrow \mathbb{R}_{\geq 0}$ is said to be of class $\mathcal{P}(\rho \in \mathcal{P})$ if $\rho$ is continuous $\rho(0)=0$ and $\rho(r)>0$ for all $r>0$.

We make the following standard assumptions on the setvalued map defining (3) guaranteeing existence of solutions.

Definition 2.1: A set-valued map $F: \mathbb{R}^{n} \rightrightarrows \mathbb{R}^{n}$ is said to satisfy the basic conditions on $\mathbb{R}^{n}$ if, for each $x \in \mathbb{R}^{n}$, $F(x)$ is nonempty, compact, and convex and if $F(\cdot)$ is upper semicontinuous on $\mathbb{R}^{n}$; i.e., for each $x \in \mathbb{R}^{n}$ and $\varepsilon>0$ there exists $\delta>0$ such that for all $\xi \in \mathbb{R}^{n}$ satisfying $\|x-\xi\| \leq \delta$, we have $F(\xi) \subset F(x)+B_{\varepsilon}(0)$.

Definition 2.2: A set-valued map $F: \mathbb{R}^{n} \rightrightarrows \mathbb{R}^{n}$ is locally Lipschitz on $\mathcal{O} \subset \mathbb{R}^{n}$ if, for all $x \in \mathcal{O}$, there exists a neighborhood $\mathcal{U} \subset \mathcal{O}$ of $x$ and $L>0$ such that $x_{1}, x_{2} \in \mathcal{U}$ implies $F\left(x_{1}\right) \subset F\left(x_{2}\right)+\bar{B}_{L\left\|x_{1}-x_{2}\right\|}(0)$.

We also make the following assumption on $F$ :

Assumption 2.3: For each $r>0$ there exists $M>0$ such that $\|x\|<r$ implies $\sup _{w \in F(x)}\|w\| \leq M$.

In terms of the system representation (2), Definition 2.1 and 2.2 and Assumption 2.3 are satisfied if $f$ is Lipschitz continuous with respect to $x$ and if $\mathbb{U}$ is convex and compact.

\section{B. (Nonsmooth) CLFs in the Dini sense}

With the set-valued map $F$ defined above, asymptotic controllability to the origin for (2) can be viewed as a weak form of asymptotic stability for (3).

Definition 2.4: The differential inclusion (3) is weakly $\mathcal{K} \mathcal{L}$-stable with respect to the origin if there exists $\beta \in \mathcal{K} \mathcal{L}$ such that, for each $x \in \mathbb{R}^{n}$, there exists $\phi \in \mathcal{S}(x)$ so that

$$
\|\phi(t, x)\| \leq \beta(\|x\|, t)
$$

for all $t \geq 0$
The connection of weak $\mathcal{K} \mathcal{L}$-stability and the existence of a smooth CLF is stated in the following theorem.

Theorem 2.5 (Smooth CLF): Suppose $F$ satisfies the basic conditions on $\mathbb{R}^{n}$, is locally Lipschitz on $\mathbb{R}^{n} \backslash\{0\}$, and satisfies Assumption 2.3. Then the origin of the differential inclusion (3) is weakly $\mathcal{K} \mathcal{L}$-stable if there exists a smooth CLF $V: \mathbb{R}^{n} \rightarrow \mathbb{R}_{\geq 0}\left(V \in C^{\infty}\right), \alpha_{1}, \alpha_{2} \in \mathcal{K}_{\infty}$ and $\rho \in \mathcal{P}$ such that for all $x \in \mathbb{R}^{n}$,

$$
\begin{gathered}
\alpha_{1}(\|x\|) \leq V(x) \leq \alpha_{2}(\|x\|), \text { and } \\
\min _{w \in F(x)}\langle\nabla V(x), w\rangle \leq-\rho(\|x\|) .
\end{gathered}
$$

Unfortunately Theorem 2.5 only provides a sufficient condition for weak $\mathcal{K} \mathcal{L}$-stability in terms of CLFs. For general systems (2), and in particular for the nonholonomic integrator (1), a continuously differentiable CLF fails to exist. In general, under the assumption of weak $\mathcal{K} \mathcal{L}$-stability, only a semiconcave (see Section II-C), and thus, Lipschitz continuous CLF $V$ can be expected [15].

To this end, generalized gradient definitions have been used to define CLFs; see [4] for a thorough discussion. Here we consider CLFs, as introduced by Sontag in [18], based on the Dini or directional derivative $d V$. Note that, as no converse result is possible in Theorem 2.5, to our knowledge Theorem 2.5 has not been written down in this form before. However, the proof easily follows by appealing to known results using generalized gradients as in Theorem 2.7 below.

Definition 2.6: Let the function $\varphi: \mathbb{R}^{n} \rightarrow \mathbb{R}$ be Lipschitz continuous in a neighborhood of $x \in \mathbb{R}^{n}$. Then the (lower) Dini derivative in direction $v \in \mathbb{R}^{n}$ is defined as

$$
d \varphi(x ; v)=\liminf _{t \searrow 0} \frac{\varphi(x+t v)-\varphi(x)}{t} .
$$

We point out two properties that will be important for our application of the Dini derivative in what follows:

(i) If $\varphi: \mathbb{R}^{n} \rightarrow \mathbb{R}$ is continuously differentiable on a neighborhood containing $x \in \mathbb{R}^{n}$, then

$$
d \varphi(x ; v)=\langle\nabla \varphi(x), v\rangle .
$$

(ii) A Lipschitz continuously differentiable function is continuously differentiable almost everywhere due to Rademacher's theorem.

Using an equivalent CLF definition in terms of the proximal subgradient, [15] extended Theorem 2.5 and obtained a necessary and sufficient condition relating weak $\mathcal{K} \mathcal{L}$-stability and the existence of Lipschitz continuous CLFs (see also [9], [10] for a locally Lipschitz construction directly in terms of the Dini derivative).

Theorem 2.7 (Dini CLF): Suppose $F$ satisfies the basic conditions on $\mathbb{R}^{n}$, is locally Lipschitz on $\mathbb{R}^{n} \backslash\{0\}$ and satisfies Assumption 2.3. Then the origin of the differential inclusion (3) is weakly $\mathcal{K} \mathcal{L}$-stable if and only if there exists a semiconcave (and thus Lipschitz continuous) CLF $V: \mathbb{R}^{n} \rightarrow$ $\mathbb{R}_{\geq 0}, \alpha_{1}, \alpha_{2} \in \mathcal{K}_{\infty}$ and $\rho \in \mathcal{P}$ such that for all $x \in \mathbb{R}^{n}$

$$
\begin{array}{r}
\alpha_{1}(\|x\|) \leq V(x) \leq \alpha_{2}(\|x\|), \text { and } \\
\min _{w \in F(x)} d V(x ; w) \leq-\rho(\|x\|) .
\end{array}
$$


In the sequel, we will refer to a locally Lipschitz function $V$ satisfying (4) and (5) as a CLF. For completeness, we next present the definition of semiconcavity and several properties of semiconcave functions.

\section{Semiconcave functions}

The last ingredient required prior to considering feedback stabilization of the nonholonomic integrator is the notion of semiconcavity (see [3, Chapter 2] for the results reviewed in this section). Herein, we make the standing assumption that $\mathcal{O} \subset \mathbb{R}^{n}$ is open.

Definition 2.8: A function $\varphi: \mathcal{O} \rightarrow \mathbb{R}$ is semiconcave if there exists a nondecreasing upper semicontinuous function $\kappa: \mathbb{R}_{>0} \rightarrow \mathbb{R}_{>0}$ such that $\lim _{\rho \searrow 0} \kappa(\rho)=0$ and

$$
\begin{array}{r}
\lambda \varphi(x)+(1-\lambda) \varphi(y)-\varphi(\lambda x+(1-\lambda) y) \\
\leq \lambda(1-\lambda)\|x-y\| \kappa(\|x-y\|)
\end{array}
$$

for any pair $x, y \in \mathcal{O}$ such that the segment $[x, y]$ is contained in $\mathcal{O}$ and for any $\lambda \in[0,1]$. We call $\kappa$ a modulus of semiconcavity for $\varphi$ in $\mathcal{O}$. A function $\varphi$ is called semiconvex in $\mathcal{O}$ if $-\varphi$ is semiconcave.

The following relationship between semiconcavity and locally Lipschitz was previously mentioned.

Theorem 2.9: A semiconcave function $\varphi: \mathcal{O} \rightarrow \mathbb{R}$ is locally Lipschitz continuous in the interior of $\mathcal{O}$.

The following two properties are useful in characterizing semiconcave functions.

Theorem 2.10: Let $\varphi: \mathcal{O} \rightarrow \mathbb{R}$, be continuously differentiable in $\mathcal{O}$. Then both $\varphi$ and $-\varphi$ are semiconcave in $\mathcal{O}$.

Corollary 2.11: Suppose $\varphi: \mathcal{O} \rightarrow \mathbb{R}$ and $\mathcal{O}$ is convex. If there exist $\varphi_{1}, \varphi_{2}: \mathcal{O} \rightarrow \mathbb{R}$ such that $\varphi_{1}$ is continuously differentiable, $\varphi_{2}$ is concave, and $\varphi=\varphi_{1}+\varphi_{2}$ holds, then $\varphi$ is semiconcave.

Theorem 2.10 and Corollary 2.11 help to identify semiconcave functions. An example of a semiconcave function is shown in Figure 1. Theorem 2.10 indicates that one only has to examine points where a given function is not continuously differentiable to check if the function is semiconcave. Since a semiconcave function is locally Lipschitz, and thus continuously differentiable almost everywhere, the semiconcavity properties of a Lipschitz continuous function depend on a set of measure zero. At points where the function is not continuously differentiable, according to Corollary 2.11, semiconcavity can be shown by defining an appropriate decomposition.

The qualitative difference between a Lipschitz continuous but not semiconcave CLF and a semiconcave CLF is shown in Figure 2 by means of the functions

$$
\begin{aligned}
& V_{L}(x)=\left(\left|x_{1}\right|+\left|x_{2}\right|\right)^{2}-0.9\|x\|^{2}, \\
& V_{S}(x)=\left(\left|x_{1}\right|-\left|x_{2}\right|\right)^{2}+0.5\|x\|^{2} .
\end{aligned}
$$

If $V_{L}$ and $V_{S}$ were nonsmooth CLFs, then the shape of the semiconcave CLF indicates that solutions $\phi \in \mathcal{S}(x)$ (based on control actions yielding a decrease in the CLF) propagate away from non-differentiable points. By contrast, in the non-semiconcave case, trajectories propagate towards



Fig. 1. Example of a semiconcave function (-) and a possible decomposition into a continuously differentiable function (- -) and a concave function $(\cdots)$. To decide if the function is semiconcave, only the point $x=1$ has to be investigated.

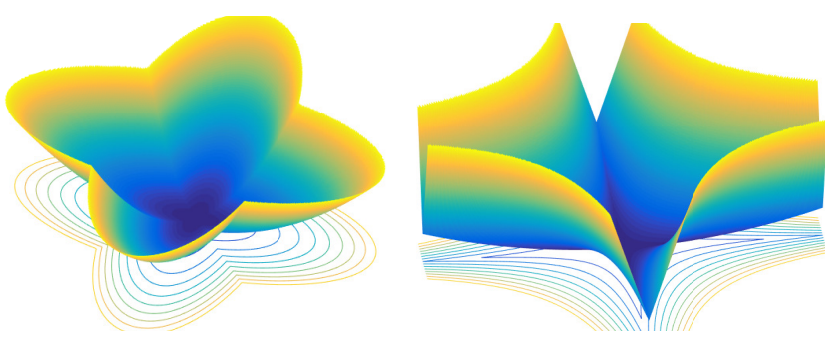

Fig. 2. Example of a semiconcave function $V_{S}$ (left) and a Lipschitz continuous non-semiconcave function $V_{L}$ (right).

non-differentiable points. One possible disadvantage of nonsemiconcave CLFs with respect to feedback design is made precise in Section III-A.

\section{FEEdBACK STABILIZATION USING CLFS}

We now describe how CLFs can be used in stabilizing feedback design for nonlinear systems. As we will use sample and hold feedbacks with piecewise constant inputs of length $\Delta T$, we begin with a definition of sample and hold feedback, stability properties in the sample and hold sense, and the connection to weak $\mathcal{K} \mathcal{L}$-stability.

Definition 3.1: For $\Delta T>0$ we call $\phi\left(\cdot, x, u_{\Delta T}\right)$ a sample and hold solution of (2) if $u_{\Delta T}: \mathbb{R}_{>0} \rightarrow \mathbb{U}$ is defined such that $u_{\Delta T}(t)=u(k \cdot \Delta T)$ holds for all $t \in[k \Delta T,(k+1) \Delta T)$ for all $k \in \mathbb{N}$. We call $\mu_{\Delta T}: \mathbb{R}^{n} \rightarrow \mathbb{U}$ a sample and hold feedback if $u_{\Delta T}(k \Delta T)=\mu_{\Delta T}(x(k \Delta T))$ holds for all $k \in$ $\mathbb{N}$. To emphasize that a particular input is a feedback, we use the notation $\phi\left(\cdot, x, \mu_{\Delta T}\right)$.

Definition 3.2 (Practical asymptotic stability): A sample and hold feedback $\mu_{\Delta T}: \mathbb{R}^{n} \rightarrow \mathbb{U}$ practically asymptotically stabilizes the origin for the dynamical system (2), if there exists a function $\beta \in \mathcal{K} \mathcal{L}$, such that for $R>\varepsilon>0$ there exists a $T_{0}>0$ such that for all $\Delta T \in\left(0, T_{0}\right]$ the solution $\phi\left(t, x, \mu_{\Delta T}\right)$ satisfies the estimate

$$
\left\|\phi\left(t, x, \mu_{\Delta T}\right)\right\| \leq \max \{\beta(t,\|x\|), \varepsilon\}
$$

for all $t \geq 0$ and for all $x \leq \bar{B}_{R}(0)$.

Theorem 3.3 ([4], Thm. 7.1): The system (3) is weakly $\mathcal{K} \mathcal{L}$-stable if and only if there exists a sample and hold feedback $\mu_{\Delta T}: \mathbb{R}^{n} \rightarrow \mathbb{U}$ which practically asymptotically stabilizes the origin for (2).

With the definition of practical asymptotic stability, we investigate three different control strategies in the remainder 
of this section.

\section{A. Steepest descent feedback}

Definition 3.4 (Steepest descent feedback): Let $V$ be a CLF for (3). We call a feedback $\mu: \mathbb{R}^{n} \rightarrow \mathbb{U}$ a steepest descent feedback if it is computed as

$$
\mu(x) \in \underset{u \in \mathbb{U}}{\operatorname{argmin}} d V(x ; f(x, u)),
$$

for all $x \neq 0$.

For a sample and hold feedback as in Definition 3.4 the following convergence result holds.

Theorem 3.5 ([4], Thm. 8.2): Let $V: \mathbb{R}^{n} \rightarrow \mathbb{R}_{\geq 0}$ be a semiconcave CLF, and let $\mu_{\Delta T}: \mathbb{R}^{n} \rightarrow \mathbb{U}$ be defined according to Definition 3.4. Then $\mu_{\Delta T}$ practically asymptotically stabilizes the origin for system (2).

Remark 3.6: The statement of Theorem 3.5 is still valid if the minimum (6) is replaced by any $\mu$ satisfying the inequality

$$
d V(x ; f(x, \mu(x)) \leq-\rho(\|x\|),
$$

where $\rho \in \mathcal{P}$ is the positive function from Theorem 2.5. This is particularly important since it implies that practical asymptotic stability can be achieved even if it is not possible to compute the global minimum of a possibly nonconvex optimization problem.

As we will see in Section IV-A, for non-semiconcave CLFs sample and hold stability does not always hold for the steepest descent closed loop system. A descriptive explanation for this property was already given in Section II-C and in Figure 2, showing that solutions propagate towards nonsmooth points of the CLF. As a consequence, semiconcavity of $V$ is crucial for Theorem 3.5 to hold. Nonetheless, it is possible to define a stabilizing feedback based on the knowledge of a non-semiconcave Lipschitz continuous CLF.

\section{B. Dini aiming}

A stabilizing sample and hold feedback based on "Dini aiming" was proposed in [9], [10]. We briefly describe the main idea here, while details can be found in [9], [10]. Let $\sigma: \mathbb{R}_{\geq 0} \rightarrow \mathbb{R}_{\geq 0}$ be a nondecreasing continuous function. The Dini aiming feedback is defined in two steps. Let $x \in \mathbb{R}^{n}$ be the current state. In the first step, the CLF $V$ is minimized over a neighborhood of $x$, i.e.,

$$
s^{\star} \in \underset{s \in \bar{B}_{r}(x)}{\operatorname{argmin}} V(s)
$$

for a given $r>0$. Based on the direction $s^{\star}$, the feedback $\mu(x)$ is defined through the optimization problem

$$
\mu(x)=\underset{u \in \mathbb{U} \cap \bar{B}_{\sigma(\|x\|+r)}(0)}{\operatorname{argmin}} \frac{\left\langle x-s^{\star}, f(x, u)\right\rangle}{\left\|x-s^{\star}\right\|}
$$

where the set of admissible control values is defined as

$$
\mathbb{U} \cap \bar{B}_{\sigma(\|x\|+r)}(0)=\{u \in \mathbb{U} \mid\|u\| \leq \sigma(\|x\|+r)\}
$$

and depends on the distance of the state to the origin. If the sampling time $\Delta T$ is chosen small enough, in accordance with $\sigma$ and $r$, the feedback practically asymptotically stabilizes the origin for system (2) (see [9], [10]). The corresponding result is summarized in the following.
Assumption 3.7: Let $V: \mathbb{R}^{n} \rightarrow \mathbb{R}_{\geq 0}$ be a Lipschitz continuous CLF. Let $\sigma: \mathbb{R}_{\geq 0} \rightarrow \mathbb{R}_{\geq 0}$ be nondecreasing and continuous, let $r>0$, and assume that for all $R>\varepsilon>0$ there exists a $c>0$ such that

$$
\min _{u \in \mathbb{U} \cap \bar{B}_{\sigma(\|x\|)}(0)} d V(x ; f(x, u))<-c
$$

for all $x \in \bar{B}_{R}(0) \cap \bar{B}_{\varepsilon}(0)$.

Theorem 3.8: Let Assumption 3.7 hold and let $\mu_{\Delta T}$ : $\mathbb{R}^{n} \rightarrow \mathbb{U}$ be defined through (7). Then $\mu_{\Delta T}$ practically asymptotically stabilizes the origin for system (2).

Compared to the steepest descent feedback, observe that for Dini aiming the CLF does not need to be semiconcave to obtain practical asymptotic stability.

Note that a function $\sigma$ satisfying the decrease condition (8) always exists since $V$ is a CLF. Because $\rho(\|x\|)>0$ for all $x \neq 0$, the minimum of $\rho(\|x\|)$ over the compact set $\bar{B}_{R}(0) \cap \bar{B}_{\varepsilon}(0)$ is attained and can be used to define $c$. If possible, an appropriate choice of $\sigma$ can ensure that $\|x\| \rightarrow 0$ implies $\|u\| \rightarrow 0$.

\section{Optimization-based feedback}

Instead of using the pointwise decrease condition (6) one can also directly minimize the Dini derivative of a given CLF in $x$ over the set of admissible constant inputs; i.e.,

$$
\min _{u \in \mathbb{U}} \int_{0}^{\Delta T} d V(\phi(t, x, u) ; f(x, u)) d t
$$

or equivalently

$$
\min _{u \in \mathbb{U}}[V(\phi(\Delta T, x, u))-V(x)] .
$$

Thus we define the one step optimization-based feedback as

$$
\mu_{\Delta T}(x) \in \underset{u \in \mathbb{U}}{\operatorname{argmin}} V(\phi(\Delta T, x, u)) .
$$

This approach implies that the solution $\phi(t, x, u)$ has to be computed over the sampling period $[0, \Delta T]$ at every time step. Observe that for some $t \in[0, \Delta t]$ it is possible that

$$
d V(\phi(t, x, \mu(x)), f(\phi(t, x, \mu(x)), \mu(x))>0 .
$$

The optimization-based feedback (9) combines the two steps of the Dini aiming (7) in a single optimization problem. Consequently, the decrease in $V$ obtained by Dini aiming provides a lower bound for the decrease in $V$ obtained by the optimization-based feedback. Thus, if Assumption 3.7 holds, it follows immediately that the optimization-based feedback practically asymptotically stabilizes the origin of (2).

Theorem 3.9: Let Assumption 3.7 hold and let $\mu_{\Delta T}$ : $\mathbb{R}^{n} \rightarrow \mathbb{U}$ be defined by (9). Then $\mu_{\Delta T}$ practically asymptotically stabilizes the origin for system (2).

Additionally observe that the optimization-based feedback explicitly depends on $\Delta T$ in contrast to the feedbacks proposed in Section III-A and III-B.

\section{FEEDBACK STABILIZATION OF THE NONHOLONOMIC INTEGRATOR}

In this section we compare the performance of the three feedback design approaches introduced in the last section 
using the dynamics of the nonholonomic integrator

$$
\left(\begin{array}{ccc}
\dot{x}_{1} & \dot{x}_{2} & \dot{x}_{3}
\end{array}\right)^{T}=\left(\begin{array}{lll}
u_{1} & u_{2} & x_{1} u_{2}-x_{2} u_{1}
\end{array}\right)^{T}
$$

with the convex and compact input set given by $u \in \mathbb{U}=$ $[-1,1]^{2}$.

We define a semiconcave CLF and a locally Lipschitz (but not semiconcave) CLF, respectively, for the nonholonomic integrator by

$$
\begin{aligned}
V_{S}(x) & =\left(\sqrt{x_{1}^{2}+x_{2}^{2}}-|x|\right)^{2}+x_{3}^{2} \\
& =x_{1}^{2}+x_{2}^{2}+2 x_{3}^{2}-2\left|x_{3}\right| \sqrt{x_{1}^{2}+x_{2}^{2}}
\end{aligned}
$$

and

$$
\begin{aligned}
V_{L}(x) & =\left(\left|x_{1}\right|-\left|x_{3}\right|\right)^{2}+\left(\left|x_{2}\right|-\left|x_{3}\right|\right)^{2}+10\left|x_{3}\right| \\
& =x_{1}^{2}+x_{2}^{2}+2 x_{3}^{2}+\left|x_{3}\right|\left(10-2\left(\left|x_{1}\right|+\left|x_{2}\right|\right)\right) .
\end{aligned}
$$

The function $V_{S}$ was shown to be a global semiconcave CLF for the nonholonomic integrator in [4]. That $V_{S}$ is semiconcave can be seen by decomposing $V_{S}$ into a continuously differentiable part $x_{1}^{2}+x_{2}^{2}+2 x_{3}^{2}$ and a concave part $-2 \sqrt{x_{1}^{2}+x_{2}^{2}}\left|x_{3}\right|$.

The function $V_{L}$ is a Lipschitz continuous (local) CLF on the domain $\mathbb{X}=[-2,2]^{3}$. A proof is provided in the Appendix. The function $V_{L}$ is not semiconcave since, for fixed $x_{1}, x_{2}$, and $10-2\left(\left|x_{1}\right|+\left|x_{2}\right|\right)>0$, it is strictly convex in a neighborhood around $x_{3}=0$. Yet, $V_{L}$ is also not semiconvex due to the strictly concave terms $-2\left|x_{1}\right|$ and $-2\left|x_{2}\right|$ in a neighborhood of the origin and fixed $x_{3} \neq 0$.

For the following numerical simulations we use the initial value of $x=(111)^{T}$ if not explicitly stated otherwise. As a stepsize for the piecewise constant input we use $\Delta T=0.02$.

The optimization problems involved in the feedback design are solved using fmincon in Matlab. Since the CLFs are nonsmooth and the dynamics are nonlinear, it is not guaranteed that a global optimal solution was found in every iteration in the following simulations.

\section{A. Numerical simulations: The steepest descent feedback}

Of the three methods, the steepest descent feedback described in Section III-A is the easiest method to implement. At every time step the optimization problem (6) is solved in order to obtain the feedback law $\mu_{\Delta T}$. The numerical results are visualized in Figure 3 and Figure 4. As one could expect from Theorem 3.5, the semiconcave CLF $V_{S}$ brings the initial state to a neighborhood of the origin (see Figure 3). By
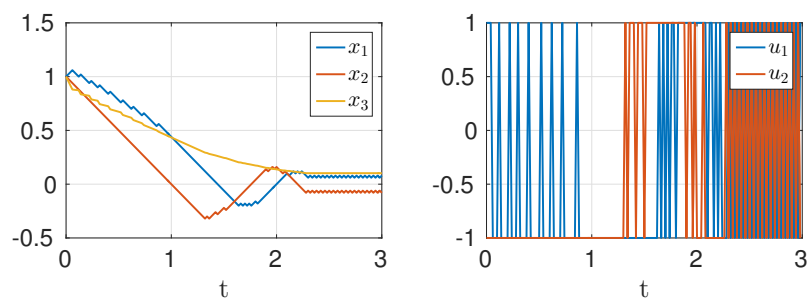

Fig. 3. Closed loop simulation using the CLF $V_{S}$ and the steepest descent feedback (6) with $\Delta T=0.02$. The feedback drives the initial state to a neighborhood of the origin which depends on the stepsize $\Delta T$. contrast, since the CLF $V_{L}$ is not semiconcave, convergence of the solution $\phi\left(t, x, \mu_{\Delta T}\right)$ to a neighborhood of the origin cannot be expected and cannot be observed in our numerical simulations (see Figure 4).


Fig. 4. Closed loop simulation using the CLF $V_{L}$ and the steepest descent feedback (6) with $\Delta T=0.02$. The solution starts to chatter after a neighborhood is reached where the function $V_{L}$ is not semiconcave.

Since (6) does not penalize $\left\|\mu_{\Delta T}\right\|$, the steepest descent feedback leads to a bang-bang control and makes it necessary that $\mathbb{U}$ is bounded. Nevertheless, the steepest descent feedback is very easy to implement and leads to practical asymptotic stability when used with a semiconcave CLF.

\section{B. Numerical simulations: The Dini aiming feedback}

In Figure 5, the numerical results of the Dini aiming feedback using the CLF $V_{S}$ are visualized. Here, the search
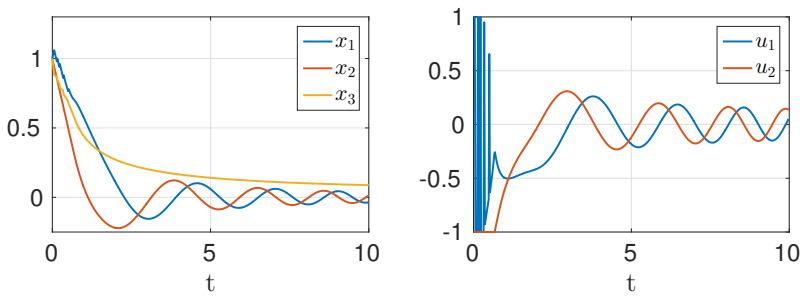

Fig. 5. Closed loop simulation using the CLF $V_{S}$ and Dini aiming with $\Delta T=0.02$. Due to the choice $\sigma(x)=\|x\|$, Dini aiming does not lead to a bang-bang control, in contrast to steepest descent.

area is defined by setting $r=0.05$. Observe that the choice of the function $\sigma(x)=\|x\|$ ensures that $\left\|\mu_{\Delta T}(x)\right\| \leq\|x\|+r$ for all $x$. The degree of freedom in the function $\sigma$ enables Dini aiming to avoid bang-bang behavior of the input $u$.

In Figure 6, the closed loop solution using the CLF $V_{L}$ is visualized. Here, we use $\sigma(x)=0.5\|x\|$. In contrast to


Fig. 6. Closed loop simulation using the CLF $V_{L}$ and Dini aiming with $\Delta T=0.02$ and $\sigma(x)=0.5\|x\|$.

steepest descent, Dini aiming stabilizes the initial value also with the function $V_{L}$. The difficulty with the Dini aiming algorithm is to define the constant $r>0$ and the function $\sigma$ in line with the step size $\Delta T$ such that a decrease in the CLF $V$ is guaranteed in every sampling period. 
For the initial value $x=(111)^{T}$ the semiconcavity of the function $V_{S}$ seems to be a disadvantage in the Dini aiming algorithm since it leads to oscillations of the solution $\phi\left(t, x, \mu_{\Delta T}\right)$. With the function $V_{L}$, the solution $\phi\left(t, x, \mu_{\Delta T}\right)$ converges to a subset of $\mathbb{R}^{3}$ on which the CLF $V_{L}$ is semiconvex. (The function $V_{L}$ is semiconvex around a neighborhood of $x_{3}=0$ excluding a neighborhood around the origin.) After the neighborhood of $x_{3}=0$ is reached, the solution $\phi\left(t, x, \mu_{\Delta T}\right)$ stays in that neighborhood and oscillations are prevented.

\section{Numerical simulations: The optimization-based feedback}

The results of the optimization-based feedback for the initial value $x=(111)^{T}$ are visualized in Figures 7 and 8. As argued in Section III-C the decrease of $V$ for
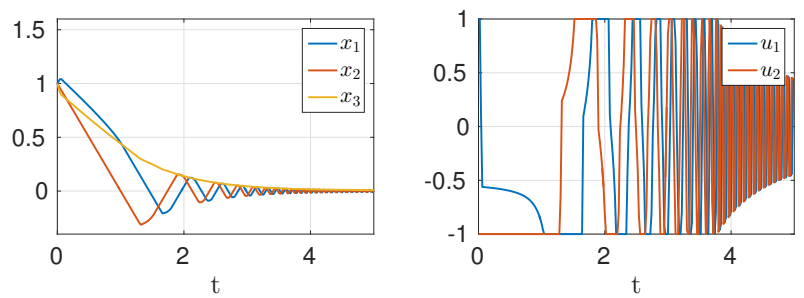

Fig. 7. Closed loop simulation using the CLF $V_{S}$ and the optimizationbased feedback with $\Delta T=0.02$.
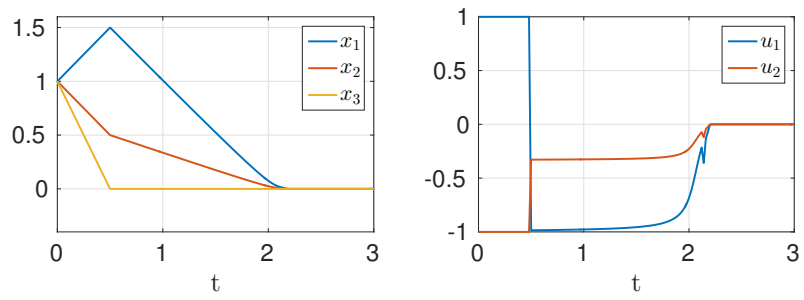

Fig. 8. Closed loop simulation using the CLF $V_{L}$ and the optimizationbased feedback with $\Delta T=0.02$.

the optimization-based feedback is at least as fast as the decrease of $V$ for the Dini aiming feedback. Thus, we obtain similar convergence results for both methods. However, the optimization-based feedback outperforms Dini aiming, since it does not depend on the choice of the search area $r>0$ or the function $\sigma$. Of course this gain of performance comes with the price of the higher computational effort for solving the underlying optimization problem.

Again, looking at the closed loop solutions using the CLF $V_{S}$ and the CLF $V_{L}$, semiconcavity seems to be a disadvantage and semiconvexity seems to be an advantage in the optimization-based feedback. In Figure 9, the closed loop solution using $V_{L}$ for the initial value $x=\left(\begin{array}{llll}0 & 0 & 0.5\end{array}\right)^{T}$ is visualized. In a neighborhood of the initial state $V_{L}$ is semiconcave and not semiconvex which leads to zigzagging. Looking at Figures 8 and 9 this raises the question if semiconvex CLFs are beneficial compared to semiconcave CLFs when an optimization-based feedback is used. Additionally, this leads to the question of existence of nonsmooth semiconvex CLFs for weakly $\mathcal{K} \mathcal{L}$-stable systems.
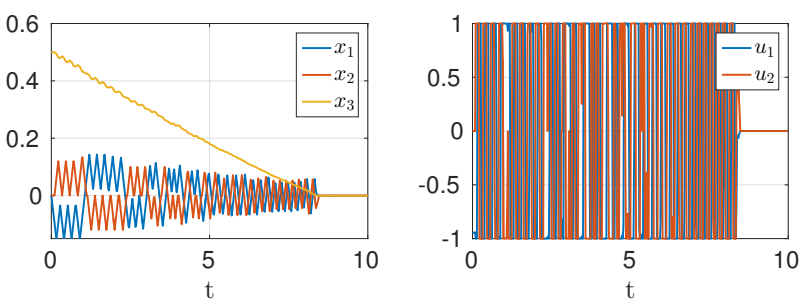

Fig. 9. Closed loop simulation using the CLF $V_{L}$ and optimization-based feedback with $\Delta T=0.02$ for the initial value $x=\left(\begin{array}{lll}0 & 0 & 0.5\end{array}\right)^{T}$.

\section{Conclusions \& Outlook}

In this paper we reviewed theoretical results for CLFs and numerical methods for feedback design using nonsmooth CLFs. To investigate the discrepancy between theory and application, a numerical case study on the example of the dynamics of the nonholonomic integrator was performed. The numerical results show the potential of CLF based feedbacks which we will further analyze by a thorough comparison with model predictive control in future work.

\section{APPENDIX}

In this section we provide a proof that the function

$$
\begin{aligned}
V_{L}(x) & =\left(\left|x_{1}\right|-\left|x_{3}\right|\right)^{2}+\left(\left|x_{2}\right|-\left|x_{3}\right|\right)^{2}+10\left|x_{3}\right| \\
& =x_{1}^{2}+x_{2}^{2}+2 x_{3}^{2}+\left|x_{3}\right|\left(10-2\left(\left|x_{1}\right|+\left|x_{2}\right|\right)\right)
\end{aligned}
$$

introduced in Equation (11) is a control Lyapunov function in the Dini sense on the domain $x \in \mathbb{X}=[-2,2]^{3}$.

As a first step, observe that with

$$
\alpha_{1}(s) \doteq s^{2} \quad \text { and } \quad \alpha_{2}(s) \doteq 2 s^{2}+10 s
$$

for all $s \in \mathbb{R}_{\geq 0}$, the inequality (4) holds for all $x \in \mathbb{X}$. The main step, showing that the decrease condition

$$
\min _{u \in \mathbb{U}} d V_{L}(x ; f(x, u)) \leq-\rho(\|x\|)
$$

is satisfied for a positive definite function $\rho \in \mathcal{P}$ for all $x \in \mathbb{X}$, will be split into two parts. (Note that the condition $u \in \mathbb{U}$ is stronger than the condition $w \in F(x)$ used in (5) as we do not need to consider the closed convex hull of the vector field to obtain suitable controls.)

\section{A. The differentiable domain of $V_{L}$}

We define the set $\mathbb{X}_{0}=\left\{x \in \mathbb{X} \mid x_{1} \neq 0, x_{2} \neq 0, x_{3} \neq 0\right\}$. If $x \in \mathbb{X}_{0}$, then $V_{L}$ is continuously differentiable. In this case, the gradient is given by

$$
\nabla V_{L}(x)=\left(\begin{array}{c}
2 x_{1}-2\left|x_{3}\right| \operatorname{sign}\left(x_{1}\right) \\
2 x_{2}-2\left|x_{3}\right| \operatorname{sign}\left(x_{2}\right) \\
4 x_{3}+\left(10-2\left(\left|x_{1}\right|+\left|x_{2}\right|\right)\right) \operatorname{sign}\left(x_{3}\right)
\end{array}\right)
$$

and thus the directional derivative can be written as

$$
\begin{aligned}
\left\langle\nabla V_{L}(x), f(x, u)\right\rangle \\
=\left[2 x_{1}-2\left|x_{3}\right| \operatorname{sign}\left(x_{1}\right)\right] u_{1} \\
\quad-\left[4 x_{3}+\left(10-2\left(\left|x_{1}\right|+\left|x_{2}\right|\right)\right) \operatorname{sign}\left(x_{3}\right)\right] x_{2} u_{1} \\
\quad+\left[2 x_{2}-2\left|x_{3}\right| \operatorname{sign}\left(x_{2}\right)\right] u_{2} \\
\quad+\left[4 x_{3}+\left(10-2\left(\left|x_{1}\right|+\left|x_{2}\right|\right)\right) \operatorname{sign}\left(x_{3}\right)\right] x_{1} u_{2} .
\end{aligned}
$$


Using the input

$$
\begin{aligned}
\tilde{u}_{1}=- & {\left[2 x_{1}-2\left|x_{3}\right| \operatorname{sign}\left(x_{1}\right)\right] } \\
& +\left[4 x_{3}+\left(10-2\left(\left|x_{1}\right|+\left|x_{2}\right|\right)\right) \operatorname{sign}\left(x_{3}\right)\right] x_{2} \\
\tilde{u}_{2}=- & {\left[2 x_{2}-2\left|x_{3}\right| \operatorname{sign}\left(x_{2}\right)\right] } \\
& -\left[4 x_{3}+\left(10-2\left(\left|x_{1}\right|+\left|x_{2}\right|\right)\right) \operatorname{sign}\left(x_{3}\right)\right] x_{1}
\end{aligned}
$$

and

$$
u_{i}(x)=\left\{\begin{array}{ccc}
\tilde{u}_{i}(x) & \text { for } & \left|\tilde{u}_{i}(x)\right| \leq 1 \\
\tilde{u}_{i}(x) /\left|\tilde{u}_{i}(x)\right| & \text { for } & \left|\tilde{u}_{i}(x)\right|>1
\end{array}\right.
$$

for $i=1,2$ shows that

$$
\min _{u \in \mathbb{U}}\left\langle\nabla V_{L}(x), f(x, u)\right\rangle \leq-u_{1} \tilde{u}_{1}-u_{2} \tilde{u}_{2} \leq 0
$$

holds for all $x \in \mathbb{X}_{0}$. As a next step we show that the decrease condition (5) is satisfied for every orthant by considering different cases. We begin with $x \in \mathbb{X} \backslash\{0\}$ and $x_{i} \geq 0, i=1,2,3$. Then

$$
\begin{aligned}
& \tilde{u}_{1}^{2}=\left(2 x_{1}-2 x_{3}-\left(4 x_{3}+\left(10-2\left(x_{1}+x_{2}\right)\right) x_{2}\right)^{2}\right. \\
& \tilde{u}_{2}^{2}=\left(2 x_{2}-2 x_{3}+\left(4 x_{3}+\left(10-2\left(x_{1}+x_{2}\right)\right) x_{1}\right)^{2}\right.
\end{aligned}
$$

holds. Since, $4 x_{3}+10-2\left(x_{1}+x_{2}\right) \geq 2$ we observe from Equation (13) that

$$
2 x_{2}-2 x_{3}+2 x_{1} \leq 0
$$

(and $-2 x_{3}+6 x_{1} \leq 0$ in the case $x_{2}=0$ ) needs to hold in order to obtain $u_{2}^{2}=0$. Inequality (14) implies that $2 x_{1}-$ $2 x_{3} \leq 0$ (or $6 x_{1}-2 x_{3} \leq 0$ in the case $x_{2}=0$ ). Combining this result with $4 x_{3}+\left(10-2\left(x_{1}+x_{2}\right)\right)>0$ in Equation (12) leads to $\tilde{u}_{1}<0$ for $x \in \mathbb{X} \backslash\{0\}$, i.e.,

$$
u_{1} \tilde{u}_{1}+u_{2} \tilde{u}_{2}>0
$$

for all $x \in \mathbb{X} \backslash\{0\}$ with $x_{i} \geq 0, i=1,2,3$. The other orthants can be iteratively reduced to the case $x_{i} \geq 0, i=1,2,3$ by using the following transformations.

- Let $-\tilde{x}_{1}=x_{1}>0, x_{2}>0$ and $x_{3}>0$ :

$$
\begin{aligned}
& \tilde{u}_{1}^{2}=\left(-2 \tilde{x}_{1}+2 x_{3}+\left(4 x_{3}+\left(10-2\left(\tilde{x}_{1}+x_{2}\right)\right) x_{2}\right)^{2}\right. \\
& \tilde{u}_{2}^{2}=\left(2 x_{2}-2 \tilde{x}_{3}+\left(4 x_{3}+\left(10-2\left(\tilde{x}_{1}+x_{2}\right)\right) \tilde{x}_{1}\right)^{2}\right.
\end{aligned}
$$

- Let $x_{1}>0,-\tilde{x}_{2}=x_{2}>0$ and $x_{3}>0$ :

$$
\begin{aligned}
& \tilde{u}_{1}^{2}=\left(2 x_{1}-2 x_{3}+\left(4 x_{3}+\left(10-2\left(x_{1}+\tilde{x}_{2}\right)\right) \tilde{x}_{2}\right)^{2}\right. \\
& \tilde{u}_{2}^{2}=\left(-2 \tilde{x}_{2}+2 \tilde{x}_{3}+\left(4 x_{3}+\left(10-2\left(x_{1}+\tilde{x}_{2}\right)\right) x_{1}\right)^{2}\right.
\end{aligned}
$$

- Let $x_{1}>0, x_{2}>0$ and $-\tilde{x}_{3}=x_{3}<0$ :

$$
\begin{aligned}
& \tilde{u}_{1}^{2}=\left(2 x_{1}-2 \tilde{x}_{3}+\left(4 \tilde{x}_{3}+\left(10-2\left(x_{1}+x_{2}\right)\right) x_{2}\right)^{2}\right. \\
& \tilde{u}_{2}^{2}=\left(2 x_{2}-2 \tilde{x}_{3}-\left(4 \tilde{x}_{3}+\left(10-2\left(x_{1}+x_{2}\right)\right) x_{1}\right)^{2}\right.
\end{aligned}
$$

Since we included $x_{1}=0, x_{2}=0, x_{3}=0$ but $x \neq 0$ in our calculations, it holds that

$$
\min _{u \in \mathbb{U}}\left\langle\nabla V_{L}(x), f(x, u)\right\rangle<0
$$

for all $x \in \mathbb{X}_{0} \cap \bar{B}_{r}(0)$ for all $r>0$, i.e., the decrease condition (5) is satisfied for all $x \in \mathbb{X}_{0}$.

\section{B. The decrease condition of $V_{L}$ using the Dini derivative}

In this section we show that the decrease condition is also satisfied at the points $x \in \mathbb{X}$ where the function $V_{L}$ is not continuously differentiable. In order to compute the Dini derivative of $V_{L}$, we first compute the Dini derivative of two auxiliary functions.

- Consider the function $\varphi: \mathbb{R} \rightarrow \mathbb{R}$ defined as $\varphi(x)=$ $c|x|$ for $c \in \mathbb{R}$. At $x=0$ the Dini derivative is given by

$$
d \varphi(0 ; v)=\liminf _{t \searrow 0} \frac{\varphi(t v)}{t}=\liminf _{t \searrow 0} \frac{c|t v|}{t}=c|v|
$$

- Consider the function $\varphi: \mathbb{R}^{2} \rightarrow \mathbb{R}$ defined as $\varphi(x)=$ $\left|x_{1}\right| \cdot\left|x_{2}\right|$. In the case $x_{1}=0$ and $x_{2} \neq 0$ we obtain

$$
\begin{aligned}
d \varphi(x ; v) & =\liminf _{t \searrow 0} \frac{\left|t v_{1}\right| \cdot\left|x_{2}+t v_{2}\right|}{t} \\
& =\liminf _{t \searrow 0}\left|v_{1}\right| \cdot\left|x_{2}+t v_{2}\right|=\left|v_{1}\right| \cdot\left|x_{2}\right| .
\end{aligned}
$$

Since $\varphi$ is symmetric, in the case $x_{1} \neq 0$ and $x_{2}=0$ we obtain

$$
d \phi(x ; v)=\left|v_{2}\right| \cdot\left|x_{1}\right| .
$$

For $x=0$ the Dini derivative satisfies

$$
\begin{aligned}
d \phi(x ; v) & =\liminf _{t \searrow 0} \frac{\left|t v_{1}\right| \cdot\left|t v_{2}\right|}{t} \\
& =\liminf _{t \searrow 0}\left|v_{1}\right| \cdot\left|t v_{2}\right|=0 .
\end{aligned}
$$

To show that the decrease condition is satisfied for the remaining set of measure zero we use the results from the auxilary functions and consider different cases.

(i) In the case $x_{1} \neq 0, x_{2}=0, x_{3}=0$ we have

$$
d V_{L}(x ; v)=2 x_{1} v_{1}+10\left|v_{3}\right|-2\left|v_{3}\right|\left|x_{1}\right| .
$$

Here we define $u$ such that $v_{3}=0$ holds, i.e.,

$$
0=v_{3}=x_{1} u_{2}-x_{2} u_{1}=x_{1} u_{2} .
$$

We set $\tilde{u}_{2}=u_{2}=0$ and $\tilde{u}_{1}=-x_{1}$,

$$
u_{1}=\operatorname{sign}\left(\tilde{u}_{1}\right) \min \left\{\left|\tilde{u}_{1}\right|, 1\right\} .
$$

and obtain the decrease condition

$$
d V_{L}(x, f(x, \tilde{u}))=-x_{1}^{2}=-\|x\|^{2} .
$$

(ii) For $x_{1}=0, x_{2} \neq 0, x_{3}=0$ the Dini derivative is given by

$$
d V_{L}(x ; v)=2 x_{2} v_{2}+10\left|v_{3}\right|-2\left|v_{3}\right|\left|x_{2}\right| .
$$

and we obtain

$$
d V_{L}(x, f(x, \tilde{u}))=-x_{2}^{2}=-\|x\|^{2} .
$$

analogously to case (i) by using the input $\tilde{u}_{1}=u_{1}=0$ and $\tilde{u}_{2}=-x_{2}$,

$$
u_{2}=\operatorname{sign}\left(\tilde{u}_{2}\right) \min \left\{\left|\tilde{u}_{2}\right|, 1\right\} .
$$

(iii) For $x_{1}=0, x_{2}=0, x_{3} \neq 0$ and $d V_{L}(x ; v)=4 x_{3} v_{3}+10 \operatorname{sign}\left(x_{3}\right) v_{3}-2\left|x_{3}\right|\left(\left|v_{1}\right|+\left|v_{2}\right|\right)$ we automatically have $v_{3}=0$ independent of the choice of $u_{1}$ and $u_{2}$. For $v_{1}=\tilde{u}_{1}=x_{3}, u_{1}=$ $\operatorname{sign}\left(\tilde{u}_{1}\right) \min \left\{\left|\tilde{u}_{1}\right|, 1\right\}$ and $v_{2}=u_{2}=0$ we obtain the decrease condition

$$
d V_{L}(x ; f(x, \tilde{u}))=-2 x_{3}^{2} \leq-\|x\|^{2} .
$$

(iv) For $x_{1} \neq 0, x_{2} \neq 0, x_{3}=0$ we obtain $d V_{L}(x ; v)=2\left(x_{1} v_{1}+x_{2} v_{2}\right)+10\left|v_{3}\right|-2\left|v_{3}\right|\left(\left|x_{1}\right|+\left|x_{2}\right|\right)$. 
We start with the condition

$$
0=v_{3}=x_{1} \tilde{u}_{2}-x_{2} \tilde{u}_{1}
$$

and assume that $x_{2} \geq x_{1}$ holds. (If $x_{2} \leq x_{1}$ the role of $u_{1}$ and $u_{2}$ can be changed due to symmetry.) We define

$$
\tilde{u}_{1}=\frac{x_{1}}{x_{2}} \tilde{u}_{2}
$$

then the decrease condition reads

$$
\begin{aligned}
d V_{L}(x ; f(x, \tilde{u})) & =2 \frac{x_{1}^{2}}{x_{2}} \tilde{u}_{2}+2 x_{2} \tilde{u}_{2} \\
& =2\left(\frac{x_{1}}{x_{2}} x_{1}+x_{2}\right) \tilde{u}_{2}
\end{aligned}
$$

If we define

$$
\tilde{u}_{2}=-\left(\frac{x_{1}}{x_{2}} x_{1}+x_{2}\right)
$$

the decrease condition, becomes

$$
\begin{aligned}
d V_{L}(x ; f(x, \tilde{u})) & =-2\left(\frac{x_{1}}{x_{2}} x_{1}+x_{2}\right)^{2} \\
& =-2\left(\frac{x_{1}^{4}}{x_{2}^{2}}+2 x_{1}^{2}+x_{2}^{2}\right) \\
& <-2\left(x_{1}^{2}+x_{2}^{2}\right)=-2\|x\|^{2}
\end{aligned}
$$

The bounded input $u$ is again obtained by projection of $\tilde{u}$ on $\mathbb{U}=[-1,1]^{2}$.

(v) For $x_{1} \neq 0, x_{2}=0, x_{3} \neq 0$, and hence

$$
\begin{aligned}
d V_{L}(x ; v)= & 2 x_{1} v_{1}+4 x_{3} v_{3}+10 \operatorname{sign}\left(x_{3}\right) v_{3}-2\left|x_{3}\right|\left|v_{2}\right| \\
& -2\left(\operatorname{sign}\left(x_{3}\right)\left|x_{1}\right| v_{3}+\operatorname{sign}\left(x_{1}\right)\left|x_{3}\right| v_{1}\right),
\end{aligned}
$$

we consider two cases. Assume that $2\left|x_{1}\right| \leq\left|x_{3}\right|$. Then we define $\tilde{u}_{2}=v_{2}=-\operatorname{sign}\left(x_{1}\right) / x_{3}$ (which implies $\left.v_{3}=-\left|x_{1}\right| / x_{3}\right)$ and $\tilde{u}_{1}=v_{1}=x_{1}$. Then the decrease condition satisfies

$$
\begin{aligned}
& d V_{L}(x ; f(x, \tilde{u})) \\
& =2 x_{1}^{2}-4\left|x_{1}\right|-10 \frac{\left|x_{1}\right|}{\left|x_{3}\right|}-2+2 \frac{\left|x_{1}\right|}{\left|x_{3}\right|}\left|x_{1}\right| \\
& \quad-2 \operatorname{sign}\left(x_{1}\right)\left|x_{3}\right| x_{1} \\
& \leq 2 x_{1}^{2}-4\left|x_{1}\right|-10 \frac{\left|x_{1}\right|}{\left|x_{3}\right|}-2+\left|x_{1}\right|-2 x_{1}^{2} \frac{\left|x_{3}\right|}{\left|x_{1}\right|} \\
& \leq 2 x_{1}^{2}-3\left|x_{1}\right|-10 \frac{\left|x_{1}\right|}{\left|x_{3}\right|}-2-2 x_{1}^{2} 2 \\
& =-3\left|x_{1}\right|-10 \frac{\left|x_{1}\right|}{\left|x_{3}\right|}-2-2 x_{1}^{2}
\end{aligned}
$$

In the case $2\left|x_{1}\right|>\left|x_{3}\right|$ we define $v_{3}=-x_{3}$, i.e., $\tilde{u}_{2}=v_{2}=-x_{3} / x_{1}$, and $\tilde{u}_{1}=v_{1}=-\operatorname{sign}\left(x_{1}\right)$. Then the decrease condition reads

$$
\begin{aligned}
d V_{L}(x ; f(x, \tilde{u}))= & -2\left|x_{1}\right|-4 x_{3}^{2}-10\left|x_{3}\right|-2\left|x_{3}\right| \frac{\left|x_{3}\right|}{\left|x_{1}\right|} \\
& -2\left(\operatorname{sign}\left(x_{3}\right)\left|x_{1}\right|\left(-\frac{x_{3}}{x_{1}}\right)-\left|x_{3}\right|\right) \\
< & -2\left|x_{1}\right|-4 x_{3}^{2}-10\left|x_{3}\right| \\
& +2 \operatorname{sign}\left(x_{1}\right)\left|x_{3}\right|-2\left|x_{3}\right| \\
< & -2\left|x_{1}\right|-\left(10-2 \operatorname{sign}\left(x_{1}\right)+2\right)\left|x_{3}\right| \\
\leq & -2\left|x_{1}\right|-6\left|x_{3}\right| .
\end{aligned}
$$

Again, we define $u$ as the projection of $\tilde{u}$.

(vi) For $x_{1} \neq 0, x_{2}=0, x_{3} \neq 0$, due to symmetry, the same arguments as in case (v) apply.

To obtain a positive function satisfying the decrease condition (5) we define $\rho \in \mathcal{P}$ by $\rho(0)=0$,

$$
\rho(s)=-\sup _{s=\|x\|} d V_{L}(x ; f(x, u(x))
$$

for $s>0$, and $u(x)$ as defined in Appendix A and B.

\section{REFERENCES}

[1] Z. Artstein. Stabilization with relaxed controls. Nonlinear Analysis: Theory, Methods \& Applications, 7(11):1163-1173, 1983.

[2] R. W. Brockett. Asymptotic stability and feedback stabilization. Differential Geometric Control Theory, pages 181-191, 1983.

[3] P. Cannarsa and C. Sinestrari. Semiconcave Functions, HamiltonJacobi Equations, and Optimal Control. Birkhäuser Boston, Boston, MA, 2004.

[4] F. Clarke. Lyapunov functions and discontinuous stabilizing feedback. Annual Reviews in Control, 35(1):13-33, 2011.

[5] F. H. Clarke, Y. S. Ledyaev, E. D. Sontag, and A. I. Subbotin. Asymptotic controllability implies feedback stabilization. IEEE Transactions on Automatic Control, 42:1394-1407, 1997.

[6] F. H. Clarke, Y. S. Ledyaev, and R. J. Stern. Asymptotic stability and smooth Lyapunov functions. Journal of Differential Equations, 149:69-114, 1998.

[7] G. Grimm, M. J. Messina, S. E. Tuna, and A. R. Teel. Model predictive control: for want of a local control Lyapunov function, all is not lost. IEEE Transactions on Automatic Control, 50(5):546-558, 2005.

[8] C. M. Kellett, H. Shim, and A. R. Teel. Further results on robustness of (possibly discontinuous) sample and hold feedback. IEEE Transactions on Automatic Control, 49(7):1081-1089, July 2004.

[9] C. M. Kellett and A. R. Teel. Uniform asymptotic controllability to a set implies locally Lipschitz control-Lyapunov function. In IEEE Conference on Decision and Control, volume 4, pages 3994-3999, 2000.

[10] C. M. Kellett and A. R. Teel. Weak converse Lyapunov theorems and control-Lyapunov functions. SIAM Journal on Control and Optimization, 42(6):1934-1959, 2004.

[11] S. Kimura, H. Nakamura, and Y. Yamashita. Control of twowheeled mobile robot via homogeneous semiconcave control lyapunov function. In Proceedings of the 9th IFAC Symposium on Nonlinear Control Systems, volume 46, pages 92-97. Elsevier, 2013.

[12] M. Krstić, I. Kanellakopoulos, and P. Kokotović. Nonlinear and Adaptive Control Design. John Wiley and Sons, Inc., 1995.

[13] M. A. Müller and K. Worthmann. Quadratic costs do not always work in MPC. Automatica, 2017. provisionally accepted.

[14] H. Nakamura. Generalized minimum projection method and its application to two wheeled mobile robot control. IFAC-PapersOnLine, 48(11):168-173, 2015

[15] L. Rifford. Existence of Lipschitz and semiconcave control-Lyapunov functions. SIAM Journal on Control and Optimization, 39(4):10431064, 2000.

[16] Y. Satoh, H. Nakamura, and S. Kimura. Discontinuous control of nonlinear systems with convex input constraint via locally semiconcave control Lyapunov functions. Proceedings of the 19th IFAC World Congress, 47(3):8629-8635, 2014.

[17] R. Sepulchre, M. Janković, and P. Kokotović. Constructive Nonlinear Control. Springer-Verlag, 1997.

[18] E. D. Sontag. A Lyapunov-like characterization of asymptotic controllability. SIAM Journal on Control and Optimization, 21(3):462-471, 1983.

[19] K. Worthmann, M. Mehrez, M. Zanon, G. K. I. Mann, R. G. Gosine, and M. Diehl. Model predictive control of nonholonomic mobile robots without stabilizing constraints and costs. IEEE Transactions on Control Systems Technology, 24(4), 2015. 